\title{
Rare case of two siblings with complete androgen insensitivity syndrome
}

\author{
Ambika Shankar*, Oby Nagar, Suwaram Saini, Babita
}

Department of Obstetrics and Gynaecology, SMS Medical College, Jaipur, Rajasthan, India

Received: 28 September 2021

Accepted: 08 November 2021

\section{*Correspondence:}

Dr. Ambika Shankar,

E-mail: ambika1993@gmail.com

Copyright: (C) the author(s), publisher and licensee Medip Academy. This is an open-access article distributed under the terms of the Creative Commons Attribution Non-Commercial License, which permits unrestricted non-commercial use, distribution, and reproduction in any medium, provided the original work is properly cited.

\begin{abstract}
Androgen insensitivity syndrome (AIS) also called testicular feminizing syndrome is a rare X linked disorder of sexual differentiation caused by mutation in the androgen receptor (AR) gene, which is located on the X chromosome (Xq11q12). In the reported cases, individuals with complete androgen insensitivity syndrome (CAIS) presented with a female appearance and normal breast development, absence of uterus and ovaries, bilateral undescended testis, and elevated testosterone levels. The syndrome is usually detected on evaluation of a phenotypic female with primary amenorrhea who presents for treatment of infertility. Here, we report 2 cases of CAIS in siblings 21 and 19 years of age who presented to us with primary amenorrhea. The elder sibling presented to us with primary amenorrhea, thelarche + , absent adrenarche, blind pouch of vagina, b/l inguinal masses suggestive of undescended testes, raised serum testosterone and absent uterus on USG. While the younger sibling also presented with similar findings but had history of $b / 1$ orchidectomy at the age of 14 years. Both the sisters were admitted in our unit and the elder sister underwent $b / 1$ orchidectomy and McIndoe's vaginoplasty. While the younger sister underwent McIndoe's vaginoplasty on the same day. Post-operative recovery was uneventful and they were given hormone replacement therapy (HRT). AIS is a rare X linked disease caused by mutation in the AR gene. which when diagnosed early can be treated appropriately. Precise diagnosis requires clinical, hormonal and molecular investigation and is of great importance for appropriate gender assignment and management in general. With timely gonadectomy, vaginoplasty or vaginal pouch deepening, hormone replacement and appropriate psychological support help the person live a healthy and almost normal life.
\end{abstract}

Keywords: AIS, Female with XY genotype, Vaginoplasty, Bilateral orchidectomy

\section{INTRODUCTION}

Androgen insensitivity syndrome (AIS) is a rare disease caused by mutation in the AR gene, which is located on the $\mathrm{X}$ chromosome (Xq11-q12). ${ }^{1}$ Owing to a complete or partial resistance to the actions of androgen, AIS can be categorized as mild (MAIS), partial (PAIS), or complete (CAIS). The clinical features of this syndrome range from infertility and/or adolescent gynaecomastia with a male phenotype, to inconclusive biological gender, to a female karyotype of $46 \mathrm{XY}^{2}{ }^{2}$ The prevalence of AIS has been estimated to be one case in every 20,000 to 64,000 newborn males for the complete syndrome (CAIS), and the prevalence is unknown for the partial syndrome (PAIS). ${ }^{3}$ CAIS was previously known as testicular feminization syndrome and was first fully described by John McLean Morris of Yale university in 1953. In the reported cases, individuals with CAIS presented with a female appearance and normal breast development, absence of uterus and ovaries, bilateral undescended testis, and elevated testosterone levels. It is the third most common cause of primary amenorrhea after gonadal dysgenesis and Müllerian agenesis. The syndrome is usually detected on evaluation of a phenotypic female with primary amenorrhea who presents for treatment of infertility. Precise diagnosis requires clinical, hormonal and molecular investigation and is of great importance for appropriate gender assignment and management in general. Here, we report 2 cases of CAIS in siblings 21 and 19 years of age with primary amenorrhea. Their clinical 
and pathological outcomes were collected and are presented here.

\section{CASE REPORT}

Would like to report a rare case of 2 sisters aged 21 and 19 respectively who were referred to us Bikaner and came to our OPD in Zenana hospital, Jaipur with complaints of primary amenorrhea for further evaluation.

The elder sister aged 21 years presented with primary amenorrhea with no history of cyclical abdominal pain and no history of treatment for the same. On general physical examination she was well built, $173 \mathrm{~cm}$ in height, with well-developed pair of breasts and absent axillary and pubic hair. On per abdominal examination bilateral undescended testes were palpable in the inguinal region. On pelvic examination $2 \mathrm{~cm}$ blind ended pouch of vaginal and normal female external genitalia were present. Ultrasonography of the whole abdomen and pelvis showed absent uterus and ovaries couldn't be visualized. On further hormonal analysis serum FSH-13.7 mIU/ml, serum LH-12.9 $\mathrm{mIU} / \mathrm{ml}$ and serum testosterone-937.16 $\mathrm{ng} / \mathrm{dl}$ (normal range for a male). When asked about a similar condition in any other family member they gave the history that, the younger sister had under gone inguinal hernia surgery at the age of 1.5 years and then due to acute pain abdomen underwent laparotomy under GA for b/l inguinal mass in 2016. During the operation gonads were found and the parents were told about the possibility of AIS in their daughter. Bilateral orchidectomy was done and HPE confirmed the inguinal masses to be testes. As, her elder sister also hadn't achieved menarche despite having well developed breasts by the age of 16 a similar condition was suspected in her but not worked up further.

Younger sister also has a similar presentation-primary amenorrhea, $163 \mathrm{~cm}$ in height, well developed breast and absent pubic and axillary hair, blind pouch of vagina and b/l orchidectomy scar + . Serum FSH-12 mIU/ml, serum LH-13.9 $\mathrm{mIU} / \mathrm{ml}$ and serum testosterone-2.3 $\mathrm{ng} / \mathrm{dl}$ (abnormally low). Karyotyping of both the siblings revealed $46+\mathrm{XY}$ genotype. They were advised to get $\mathrm{b} / \mathrm{l}$ orchidectomy for the elder sister as the development of secondary sexual characters were complete and vaginoplasty for both the siblings few months before assumption of sexual activity.

They got admitted in our unit in May 2021 for b/l orchidectomy and vaginoplasty for the elder sister. Vaginoplasty was planned for the younger sister (already had undergone b/l orchidectomy) and both were operated on the same day.

Orchidectomy was done by the urologist (as shown in Figure 1 and 2) followed by McIndoe's vaginoplasty (Figure 3 and 4) by us with the help of the plastic surgeon for the skin graft taken from their thigh (Figure 5-7). Post operatively recovery was uneventful. Their molds were changed 1 week post operatively and a deep vagina of 4.5 inches was achieved for the elder sister and 4 inches vaginal depth in the younger one. They were advised to continue using foam vaginal mold the keep the vagina patent till assumption of sexual activity and were put on estrogen replacement therapy.

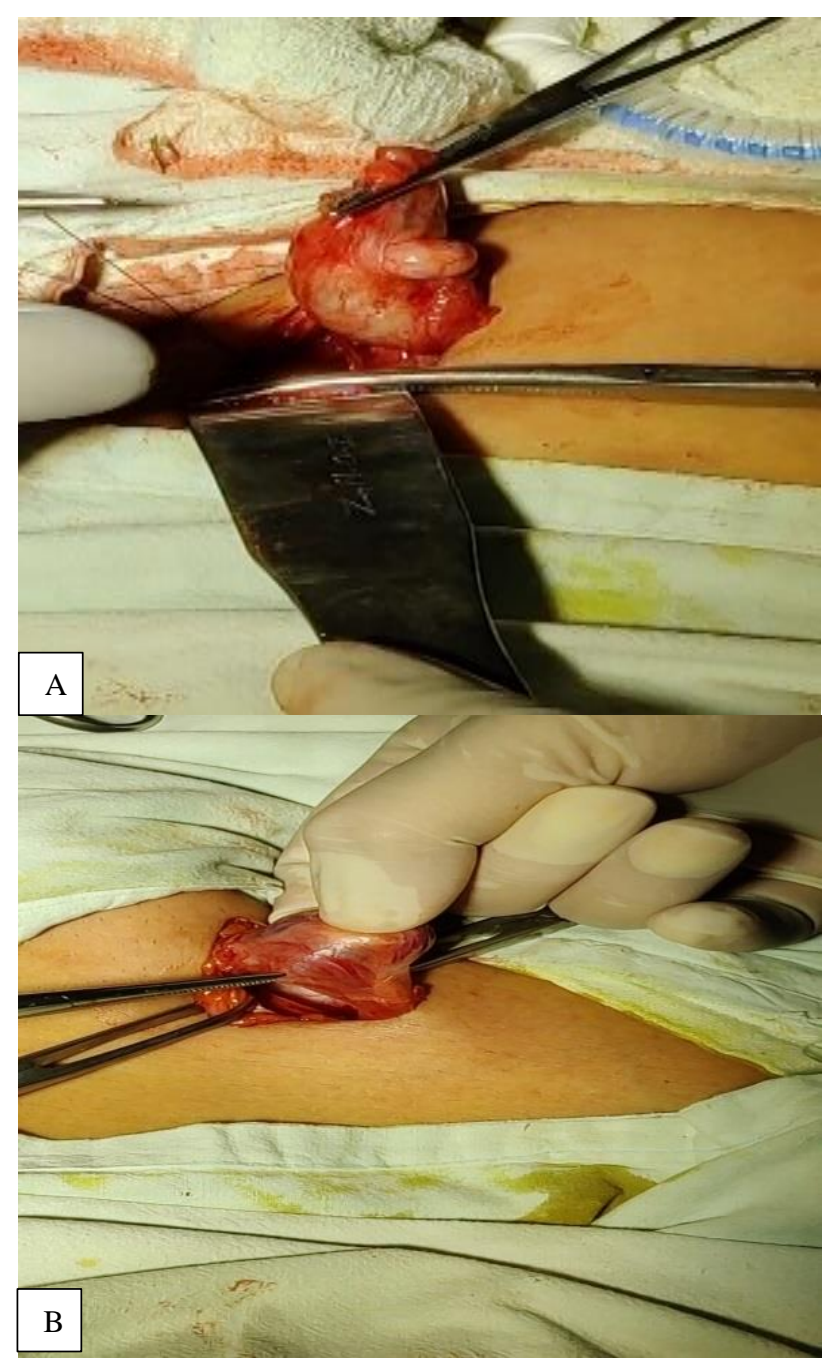

Figure 1 (A and B): Bilateral orchidectomy of undescended testis (in the inguinal canal).

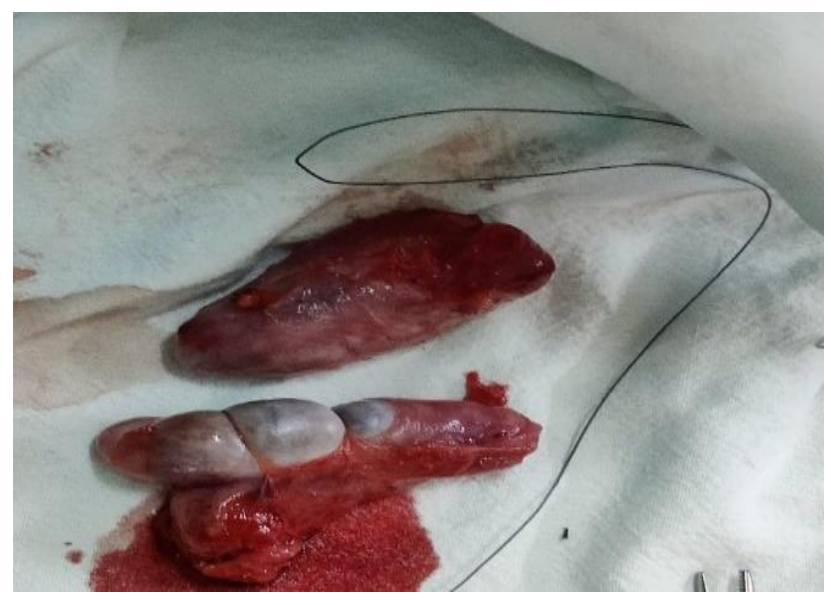

Figure 2: Bilateral orchidectomy specimen. 


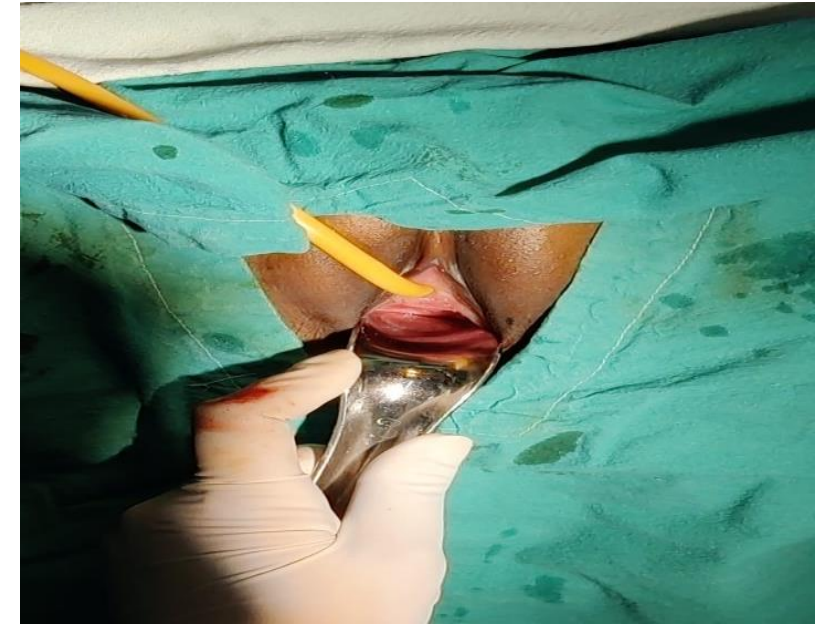

Figure 3: Blind pouch of vaginal no cervix visualized.

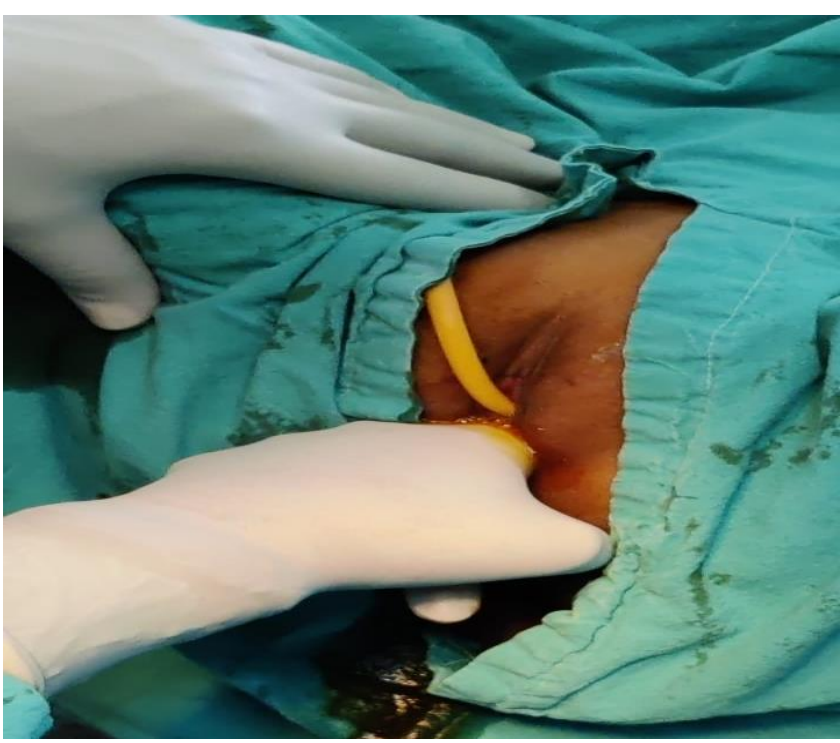

Figure 4: A cruciate incision was given and blunt dissection don't create space between the bladder and rectum.

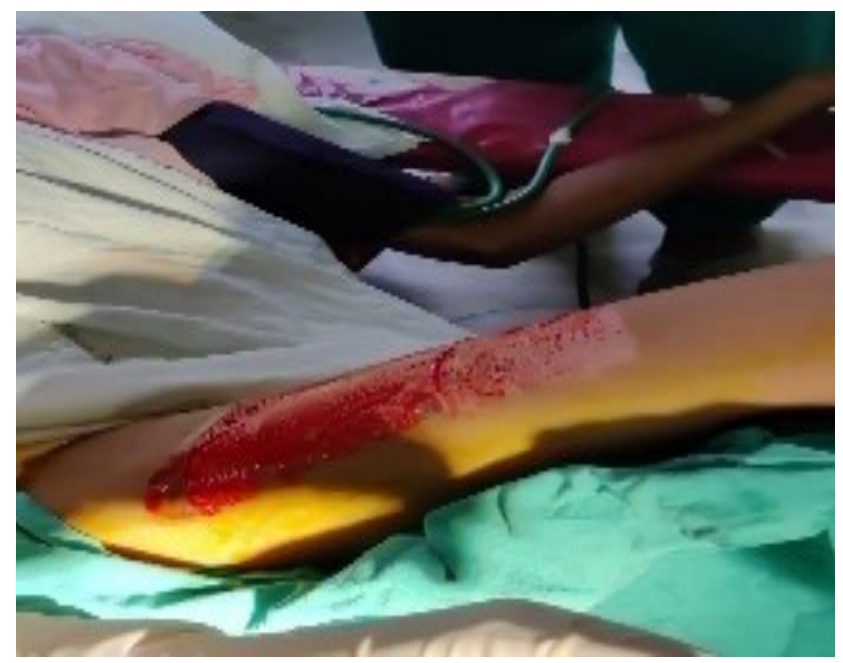

Figure 5: Split skin graft from the inner thigh.

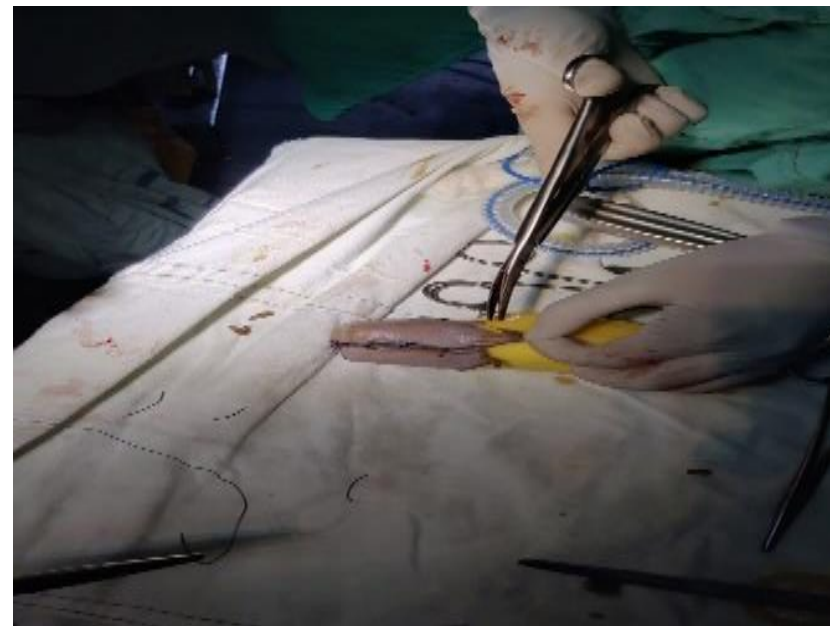

Figure 6: Stitching the split skin graft over a foam mold covered with a condom to apply over the neovagina.

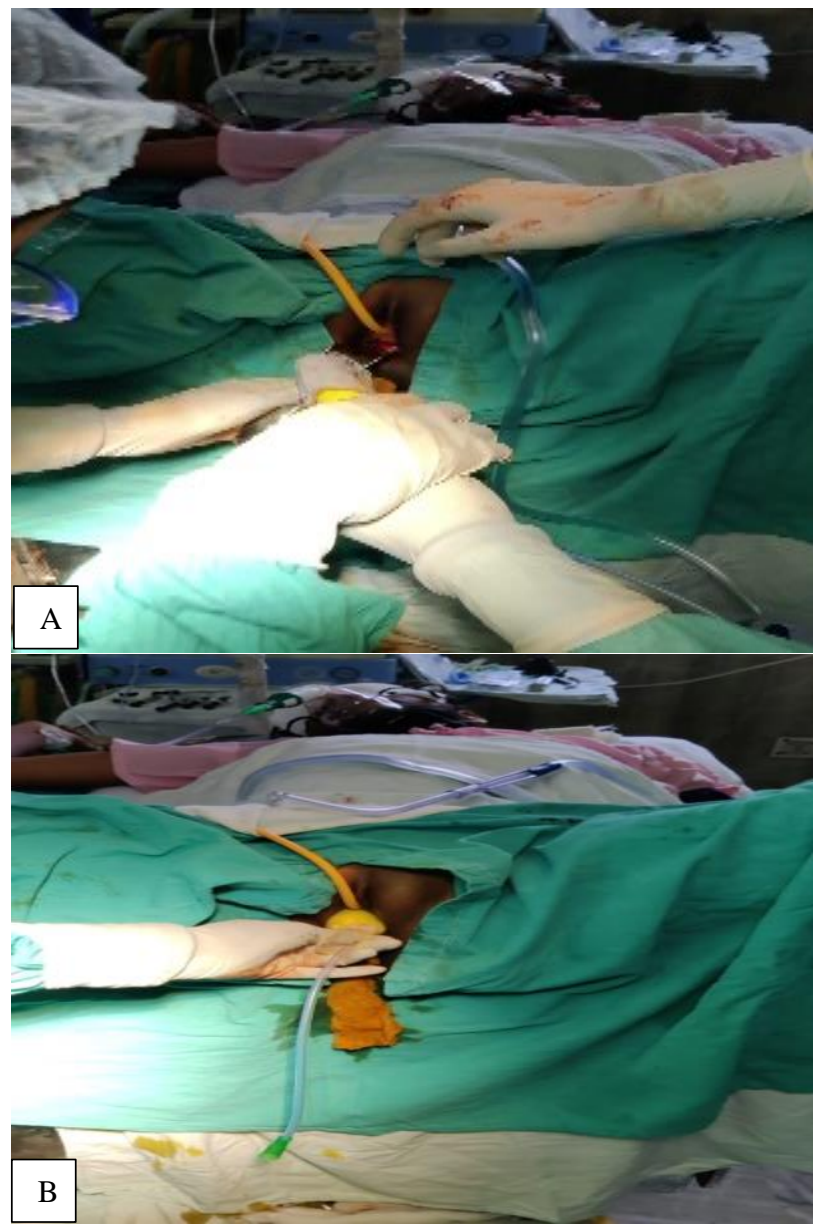

Figure 7 (A and B): Mold placed inside the neo-vagina and graft attached to the neo-vagina by anchoring sutures at 12, 3, 6 and 9 o'clock.

\section{DISCUSSION}

AIS is a type of disorder of sexual development (DSD). Generally speaking, DSD is caused by a defect in gonadal 
development, androgen synthesis, and/or the actions of androgen. Given this, AIS is classed as a defect in the actions of androgen. Although the formed testis secretes sufficient amounts of active androgen, a lack of function of the AR is the main reason for androgen insensitivity in these patients. ${ }^{4}$

The AR gene is approximately $90 \mathrm{~kb}$ in size and is located on the X chromosome (Xq11-q12). The gene has eight exons, which encode for a 917 amino acid-long protein. The AR is a member of the steroid hormone family of receptors, which also includes estrogen, progesterone, and glucocorticoid receptors. The AR contains four functional domains: A large N-terminal domain (NTD) encoded by the largest (1613 base pairs) exon 1, a DNA-binding domain (DBD) encoded by exons 2 and 3, a hinge domain, and a C-terminal ligand-binding domain (LBD) encoded by exons 5-8. The N-terminal portion is essential for its receptor activity. The DBD portion contains two zinc finger regions to both bind DNA and activate genetic transcription. The C-terminal ligand-binding domain is the most commonly reported site of AR mutations. ${ }^{5}$

AIS is a rare disease and there is no clear data regarding its prevalence. Reported estimates range from 1:20,000 to 1:64,000 genetic males with CAIS. ${ }^{3}$ AIS is classed into three subtypes: (1) MAIS, which is found in males with persistent gynecomastia and/or infertility; (2) PAIS, which is found in individuals with atypical genitalia, and (3) CAIS, which is found in individuals with a $46 \mathrm{XY}$ female karyotype. The typical presentation for CAIS includes a 46 XY karyotype with a female phenotype, primary amenorrhea in adolescence, and/or an inguinal mass in an infant. CAIS presents as an inguinal hernia or labial swelling containing a testis in an apparently female infant. In this case, the patient (younger sister) reported that the bilateral inguinal mass was discovered when she was 1.5 years a then operated upon when she was 14-years old. A female phenotype with 46-XY always has normal breast development, while pubic and axillary hair are usually absent or can be present in sparse amounts. Development of oestrogen-dependent secondary sexual characteristics occurs as the result of excess aromatisation of androgens.

The development of the uterus, cervix, and proximal vagina are suppressed in CAIS, due to the actions of antiMullerian hormone (AMH) produced by the testes. The vagina varies from a dimple in the perineum to one of normal length, but is always blind-ending. The clinical examination presented in these 2 cases fits this description. Adult women with CAIS are generally taller than women without the syndrome, but are-on average-shorter than the male population. The case we report here was also similar in this regard. Notably, it has been reported that patients who delay gonadectomy are even taller. ${ }^{6}$ The female with CAIS have normal or above-normal levels of serum testosterone and LH, while FSH levels are not increased. The reason for this may be that gonadal inhibin regulates FSH.
Bilateral gonadecomy should be performed after diagnose to avoid the risk of malignancies (gonadoblastoma in undescended testis). ${ }^{7}$ However, maintenance of the testes could maintain estrogen at a normal level to allow for spontaneous breast development. The risk of malignancies is very low before adolescence, but increases with time. Given this, the timing of the surgical removal of bilateral testes is very important. Estrogen replacement is necessary after surgery. AIS is a rare disease, however, genetic and phenotype gender separation remain problematic in these patients. Diagnosing the disease requires genetic analysis. In a recent study, it was reported that analysis of $\mathrm{AR}$ mRNA obtained from whole blood would be a potential strategy for the detection of AR gene splicing defects. The time for bilateral gonadectomy is very important and estrogen replacement is necessary. We managed the present cases with bilateral orchidectomy, McIndoe's vaginoplasty and estrogen supplementation.

AIS is associated with numerous psychosexual issues. Therefore, systematic disclosure of the diagnosis of AIS should be done in an empathic environment, with both professional and family support. The 46-XY phenotypic female is almost always infertile. However, carrier females have a $50 \%$ chance of transmitting the mutated AR gene in each pregnancy. Carrier testing is advocated within the family because the disease has known familial tendencies. These 2 cases were the only known cases in her family and no carrier testing has been done in other family members but has been advised.

\section{CONCLUSION}

AIS is a rare $\mathrm{X}$ linked disease caused by mutation in the AR gene. Which when diagnosed early can be treated appropriately with timely gonadectomy, vaginoplasty or vaginal pouch deepening, hormone replacement and help the person live a healthy and almost normal life. Detailed education regarding the disease, advice regarding testing in the other family members to identify carriers and psychological care from doctors, psychologists, and support from family are absolutely necessary in the procedures required for the treatment condition.

\section{Funding: No funding sources \\ Conflict of interest: None declared \\ Ethical approval: Not required}

\section{REFERENCES}

1. Boehmer AL, Brinkmann O, Bruggenwirth $\mathrm{H}$, Van Assendelft C, Otten BJ, Verleun-Mooijman MC et al. Genotype versus phenotype in families with androgen insensitivity syndrome. J Clin Endocrinol Metab. 2001;86(9):4151-60.

2. Danilovic DL, Correa PH, Costa EM, Melo KF, Mendonca BB, Arnhold IJ. Height and bone mineral density in androgen insensitivity syndrome with mutations in the androgen receptor gene. Osteoporos Int. 2007;18(3):369-74. 
3. Prevalence of rare diseases. Bibliographic data. Available at: http://www.orpha.net/consor/cgibin/Education_Home.php?lng=EN. Accessed on 10 Feb, 2020.

4. Edelsztein NY, Grinspon RP, Schteingart HF, Rey RA. Anti-Mullerian hormone as a marker of steroid and gonadotropin action in the testis of children and adolescents with disorders of the gonadal axis. Int $\mathbf{J}$ Pediatr Endocrinol. 2016;20.

5. Hughes IA, Deeb A. Androgen resistance. Best Pract Res Clin Endocrinol Metab. 2006;20:577-98.

6. Lanciotti L, Cofini M, Leonardi A, Bertozzi M, Penta L, Esposito S. Different Clinical Presentations and
Management in Complete Androgen Insensitivity Syndrome (CAIS). Int J Environ Res Public Health. 2019;16(7):1268.

7. Patel V, Casey RK, Gomez-Lobo V. Timing of Gonadectomy in Patients with Complete Androgen Insensitivity Syndrome-Current Recommendations and Future Directions. J Pediatr Adolesc Gynecol. 2016;29(4):320-5.

Cite this article as: Shankar A, Nagar O, Saini S, Babita. Rare case of two siblings with complete androgen insensitivity syndrome. Int J Reprod Contracept Obstet Gynecol 2021;10:4585-9. 